\title{
Human Umbilical Cord Mesenchymal Stem Cell-induced Osterix, Bone Morphogenetic Protein-2, and Tartrate-resistant Acid Phosphatase Expression in Osteoporotic Mandibular Bone
}

\author{
Nike Hendrijantini ${ }^{1}$ Cindy Karina Hartono ${ }^{1}$ Reni Puspa Daniati ${ }^{1}$ Guang Hong ${ }^{2}$ Ratri Maya Sitalaksmi ${ }^{1}$ \\ Mefina Kuntjoro ${ }^{1}$ Muhammad Dimas Aditya Ari ${ }^{1}$ \\ ${ }^{1}$ Department of Prosthodontic, Faculty of Dental Medicine, \\ Universitas Airlangga, Surabaya, Indonesia \\ ${ }^{2}$ Liaison Center for Innovative Dentistry, Graduate School of \\ Dentistry, Tohoku University, Aoba-ku, Sendai, Japan

\begin{abstract}
Address for correspondence Nike Hendrijantini, DDS, MSc, Department of Prosthodontic, Faculty of Dental Medicine, Universitas Airlangga, Mayjen Prof. Dr Moestopo 47, Surabaya 60132, Indonesia (e-mail: nike-h@fkg.unair.ac.id).
\end{abstract}

Eur J Dent:2021;5:84-89

\begin{abstract}
Keywords

- stem cells

- osteoporosis

- bone

- BMP 2

- osterix

- TRAP

- PKH-26

Objectives The aim of this study was to prove that human umbilical cord mesenchymal stem cell (hUCMSC) therapy conducted according to the mandibular osteoporotic model will increase Osterix (Osx) and bone morphogenetic protein-2 (BMP-2) expression, while reducing tartrate-resistant acid phosphatase (TRAP) expression. PKH26 labeling proves that mandibular bone regeneration is produced by hUCMSCs induction. Materials and Methods This study incorporated a true posttest only control group design. Twenty-five female Wistar rats were randomly divided into five groups consisting of the sham surgery $(N)$ group, osteoporotic groups injected with gelatin for 4 weeks (G4) and 8 weeks (G8), and osteoporotic groups injected with hUCMSC-gelatin for 4 weeks (SC4) and 8 weeks (SC8). All subjects were provided for BMP-2, Osx, and TRAP on immunohistochemistry examination and PKH-26 labeling.

Statistical Analysis All data were analyzed using ANOVA and Tukey HSD tests with $p<0.05$ being considered as statistically significant.

Results Compared with other groups, the highest level of BMP-2 and Osx occurred in the sham surgery $(N)$ and osteoporotic groups injected with hUCMSCs-gelatin (SC), while the lowest level of TRAP was found in SC4. During 4- and 8-week observation periods, the PKH 26 appeared green (fluorescent).

Conclusions hUCMSC demonstrates high-osteogenic activity and increased osteoporotic mandibular bone regeneration, as shown by increased expression of Osx and BMP-2 and decreased TRAP expression. From the labeling, PKH-26 proved that viable hUCMSCs in gelatin solvent can be present in the mandibular bone and be capable of promoting osteogenic differentiation and increasing mineralization and bone formation in the osteoporotic mandibular bone.
\end{abstract}

DoI https://doi.org/ 10.1055/s-0040-1715987 ISSN 1305-7456.
(C) 2020. European Journal of Dentistry.

This is an open access article published by Thieme under the terms of the Creative Commons Attribution-NonDerivative-NonCommercial-License, permitting copying and reproduction so long as the original work is given appropriate credit. Contents may not be used for commercial purposes, or adapted, remixed, transformed or built upon. (https://creativecommons.org/licenses/by-nc-nd/4.0/) Thieme Medical and Scientific Publishers Pvt. Ltd., A-12, 2nd Floor, Sector 2, Noida-201301 UP, India 


\section{Introduction}

Osteoporosis is a skeletal disease characterized by a total reduction in bone density due to the body's inability to manage the mineral content of the bones and damage to bone architecture, leading to reduced bone strength. ${ }^{1}$ Its complications such as osteoporotic fracture were associated with significant morbidity as well as treatment costs. ${ }^{2}$ Females are known to be more susceptible to osteoporosis than males, a situation resulting from the menopause, which induces hormonal imbalances in them, including decreased levels of estrogen which, in turn, reduce bone density. ${ }^{3}$

Osteoporosis constitutes a complication of prosthodontics-related dental treatment caused by significant bone resorption, affecting retention and stabilization of dentures. One novel, effective, and practical alternative for missing tooth replacement involves dental implants, as demonstrated by the prevalence of their use in the United States, having increased significantly from $0.7 \%$ in 2000 to $5.7 \%$ in $2016 .{ }^{4}$

One prerequisite for successful implants is the occurrence of osseointegration, which represents a clearly defined structural and functional relationship between the bone and the surface of the implant. It is necessary to observe the thickness of the jawbone in postmenopausal females before implementing prosthodontic treatment to prevent implant placement failure, thereby ensuring effective osseointegration. ${ }^{5}$

Ovariectomized rats are the most commonly used animal subjects in pathophysiological studies of osteoporosis, since they can illustrate the effect of estrogen deficiency on the decline of trabecular bone mass in humans. Osteoporosis will first attack the axial bone (mandible) and, subsequently, limb bones. It can be used as a diagnostic marker of osteoporotic abnormalities systemically. ${ }^{6}$

Stem cells constitute as yet undifferentiated immature cells which are capable of differentiating and self-renewal, depending on the potential of the tissues in which they are produced. Given the presence of a widespread defect, the number of stem cells in the body is insufficient to rapidly regenerate tissue. Therefore, a strategy for administering stem cells from outside the body is required. ${ }^{7}$

An ideal source for tissue engineering must satisfy several conditions, including ease of access, adequate cell counts, and a high-degree of immunocompatibility. An important source that has been the object of investigation for some time is that of human umbilical cord mesenchymal stem cells (hUCMSCs), the inducted form of which can assume the role of osteoprogenitors and promote bone formation. Previous research has confirmed that the application of hUCMSCs promotes high-levels of osteoblast differentiation in an osteoporotic model, a finding that is supported by increased numbers of osteoblasts and expression of transforming growth factor- $\beta 1$ (TGF- $\beta 1$ ) and Runt-related transcription factor 2 (Runx2), ${ }^{8}$ and the other research has confirmed that increased expression of ALP, type I collagen and osteocalcin, and a larger trabecular bone area in osteoporotic rats induced by hUCMSCs is the proof of increased osteoblastogenesis. ${ }^{9}$

Greater bone regeneration is characterized by an increase in bone formation markers such as bone morphogenetic protein-2 (BMP-2), Osterix (Osx) expression, and decrease in bone resorption markers like tartrate-resistant acid phosphatase (TRAP) expression. BMP-2 is a protein superfamily of transforming growth factor beta (TGF- $\beta$ ) active in osteogenesis, whereas Osx is a specific transcription factor of osteoblasts which activates gene receptors during differentiation of preosteoblasts into mature osteoblasts. ${ }^{10,11}$ TRAP is highly expressed in chondroclasts and able to degrade phosphoproteins such as osteopontin (OPN) from skeletons. A hUCMSCs-induced reduction in osteoclasts was evidenced by lower levels of TRAP expression. ${ }^{12}$ This research aims to demonstrate the ability of hUCMSCs to promote osteoporotic mandibular bone regeneration through BMP-2, Osx, and TRAP expression.

\section{Materials and Methods}

\section{Sample Preparation}

Ethical commission of health research of Universitas Airlangga Faculty of Dental Medicine has approved this study (Number: 043/HRECC.FODM/II/2019 and Number: 042/HRECC.FODM/II/2019). This research represented a true experimental study. Samples were collected from 25 three-month-old, female Wistar strain Rattus norvegicus Albinus 180 to $200 \mathrm{~g}$ in weight. Samples were randomly classified into five groups: a normal group with sham surgery $(N)$, an ovariectomy group whose members were injected with gelatin solvent for 4 weeks (G4) or 8 weeks (G8), and an ovariectomy group whose members were injected with hUCMSCs and gelatin for 4 weeks (SC4) or 8 weeks (SC8). RSUD Dr. Soetomo Surabaya (Dr. Soetomo Academic Medical Center Hospital) and the Ethical Commission of the Faculty of Veterinary Medicine, Universitas Airlangga, approved the health research ethics for the research reported here.

\section{Ovariectomy Preparation}

An ovariectomy involving the Khajuria procedure for was performed osteoporotic conditions, and sham surgery on the control group members was conducted. The subjects underwent these procedures on osteoporosis after 12 weeks. ${ }^{13}$

\section{hUCMSCs Culture Preparation}

Umbilical cords were retrieved from the healthy C-sections of newborn babies at RSUD Dr. Soetomo, Surabaya, Indonesia. The cords were then cut into $1 \mathrm{~cm}$ lengths, while the veins, arteries, and adventitia were removed to obtain Wharton's jelly, which was subsequently handled by Han's method. The medium was changed once every 3 days. $^{7}$

\section{Injection of Osteoporotic hUCMSCs into Mice Subjects and the Gelatin Solvent Procedure}

The left mandibular of the samples was penetrated through the skin under the molar region into trabecular area with a perforator needle (Stabident; Miami, USA) under anesthesia. The needle was then retrieved. Members of the G4 and G8 subject groups were injected with $50 \mu \mathrm{L}$ of gelatin solvent, while 400.000 cells in $50 \mu \mathrm{L}$ of gelatin solvent were injected into the SC4 and SC8 group subjects. 
Procedure for Injection of hUCMSCs into the Mandibular Osteoporotic Subjects Using Labeled Cells Osteoporotic subjects were prepared for injection with PKHlabeled 26 hUCMSCs before being allocated to one of two groups, two subjects to the 4-week group, and two to the 8 -week group. Four and eight weeks postinjection of hUCMSCs with PKH 26 labeling, the subjects were terminated in the same manner as those which had not been labeled.

\section{Termination of Experimental Subjects and Microscopic} Analysis of Testing Samples

Following the elapse of the testing period, termination of the research subjects was undertaken. Immunohistochemical staining using a monoclonal mouse BMP-2 (Abcam, United Kingdom), Osx (Santa Cruz Biotechnology, Inc, Europe), and TRAP (Bioss Antibodies, Netherlands) antibodies was conducted. A light microscope (Nikon H600L, Tokyo, Japan) fitted with a DS-Fi2 300 MegaPixel digital camera and a Nikon Image System photo editor program was employed for the purposes of microscopic observation. The results were measured using the Remmele immunohistochemistry index scale to count the total number of osteoblast cells (both surface and mesenchymal) from five different fields of view at 400x magnification. Fresh bone was cut with a LEICA cryocut in a dark room at a temperature of between $-40^{\circ} \mathrm{C}$ and $60^{\circ} \mathrm{C}$. PKH-26 contains fluorescent proteins which were then observed by means of a fluorescent microscope (OLYMPUS FSX-100) ${ }^{14}$ The fresh bone to be cut must be wrapped in aluminum foil and conditioned in a dark room.

\section{Statistical Analysis}

All the data was shown in five different experimental groups. Statistical analysis using the SPSS version 15.0 (SPSS, Inc.; Chicago, IL, USA), ANOVA and Tukey HSD tests was performed with the statistically significant $p<0.05$ being considered.

\section{Results}

\section{Isolation and Culture of Human Umbilical Cord Mesenchymal Stem Cells}

The isolation and culture of hUCMSCs have yet to be published. The experiment result verified that the cells were hUCMSCs by evaluating the surface marker of isolated cells, i.e., CD45-, CD73 +, CD90 +, and CD $105+$.

\section{Labeling PKH 26}

To identify the effect of hUCMSCs on the osteoporosis model, an evaluation is required to ensure that MSC inside the mandibular bone originates from exogenous hUCMSCs. At the next stage of the process, hUCMSCs labeling of each subject in the SC4 and SC8 groups using PKH26 was completed. Postinjection observation of the mandibles of the members of the group SC4 4 weeks later detected the presence of strongly fluorescent, green, labeled cells. The microscopic view of PKH26 expression can be seen in - Fig. 1.

\section{The Expression of BMP-2, Osx, and TRAP}

The microscopic expression of BMP-2 is shown in - Fig. 2 a. The number of BMP-2 expressions in each group is contained

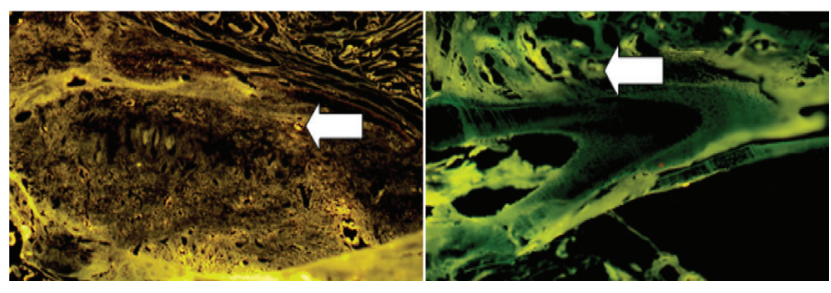

Fig. 1 Microscopic image of human umbilical cord mesenchymal stem cells (hUCMSCs) with PKH26 labeling.

in -Fig. 3. The BMP-2 normality test with KolmogorovSmirnov is normal $(p=0.950)$ and the homogeneity test with Levene statistic is homogenous $(p=0.434)$. The oneway ANOVA showed significantly difference between groups $(p=0.000)$. The Tukey HSD test showed BMP-2 expression had significantly increased in the SC4 and SC8 groups compared with the other groups. The lowest BMP-2 expression was observed in the G4 and G8 groups (SC4 $=0.041 ;$ SC8 $=0.004$ to G4) (SC8 $=0.019$ to G8).

The expression of Osx is shown in - Fig. 2 (b). The number of Osx expressions in each group is presented in - Fig. 4. The Osx normality test with Kolmogorov-Smirnov is normal $(p=0.868)$ and the homogeneity test with Levene statistic is homogenous $(p=0.138)$. The one-way ANOVA showed significantly difference between groups $(p=0.000)$. The Tukey HSD test showed Osx expression had significantly increased in the SC4 and SC8 groups compared with the other groups. The lowest Osx expression was observed in the G4 and G8 groups (SC4 $=0.010$ to G4) (SC4 $=0.002$; SC8 $=0.030$ to G8).

The expression of TRAP is shown in -Fig. 2 (c). The number of TRAP expressions in each group in contained in -Fig. 5. The TRAP normality test with Kolmogorov-Smirnov is normal ( $p=0.922)$ and the homogeneity test with Levene statistic is homogenous $(p=0.782)$. The one-way ANOVA showed significantly difference between groups $(p=0.000$ ). The Tukey HSD test showed TRAP expression was significantly reduced in the SC4 and SC8 groups compared with the other groups. The lowest TRAP expression was observed in the SC4 and SC8 groups ( $\mathrm{SC} 4=0.000$ to $\mathrm{G} 4$ and G8) (SC8 $=0.034$ to G8).

\section{Discussion}

Estrogen deficiency, resulting in postmenopausal disorders, causes a decrease in stem cells and triggers disruption in MSCs, which is the source of osteoprogenitor and osteoblasts in bone formation. ${ }^{15}$ Reduced bone density in osteoporosis occurs due to MSCs defects, leading to lower osteoblast proliferation and differentiation. ${ }^{16}$ MSCs, which are sourced from hUCMSCs, undergo several changes, transforming them into osteoprogenitors and, eventually, osteoblast cells. The differentiation between MSCs and osteoblasts includes certain growth factors as well as transcription factors such as BMP-2 and Osx.

The results of this study support those of previous research, which reported significant differences in TGF$\beta 1$ and RUNX-2 expression between the administration of hUCMSCs and osteoporosis. ${ }^{8}$ BMP-2 is known to be a cytokine group within the TGF- $\beta$ subgroup. Osx constitutes a 

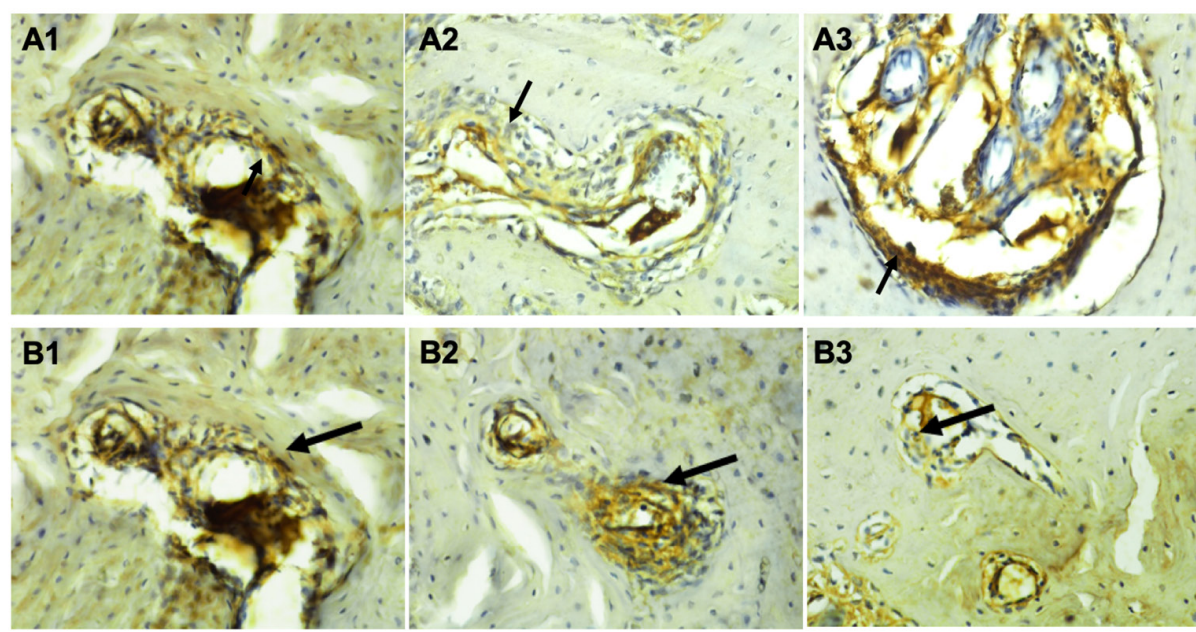

B2.

B3

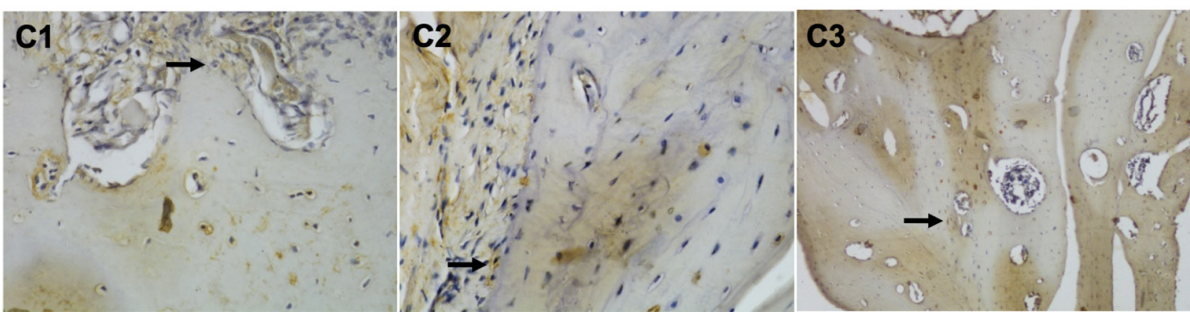

Fig. 2 Microscopic image of bone morphogenetic protein-2 (BMP-2) (a), Osterix (Osx) (b) and tartrate-resistant acid phosphatase (TRAP) (c) expression. Arrows show the expression in an immunoreactive osteogenic cell.

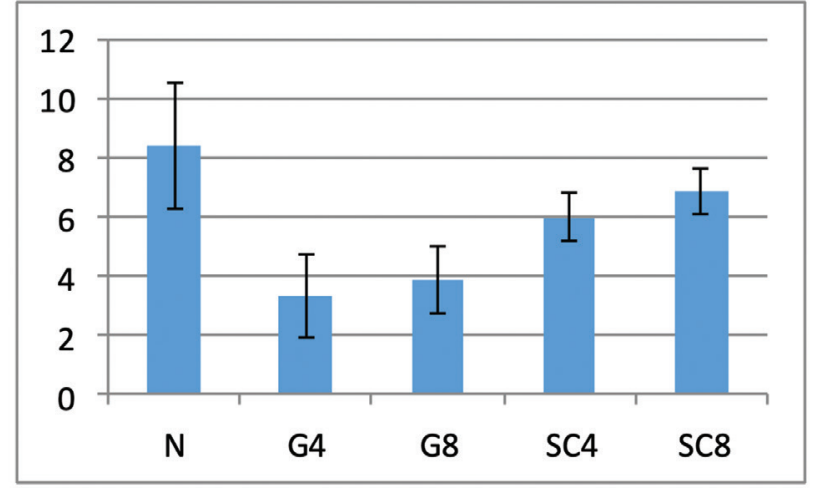

Fig. 3 Graph of mean value and standard deviation (SD) in the bone morphogenetic protein-2 (BMP-2) expression of each group.

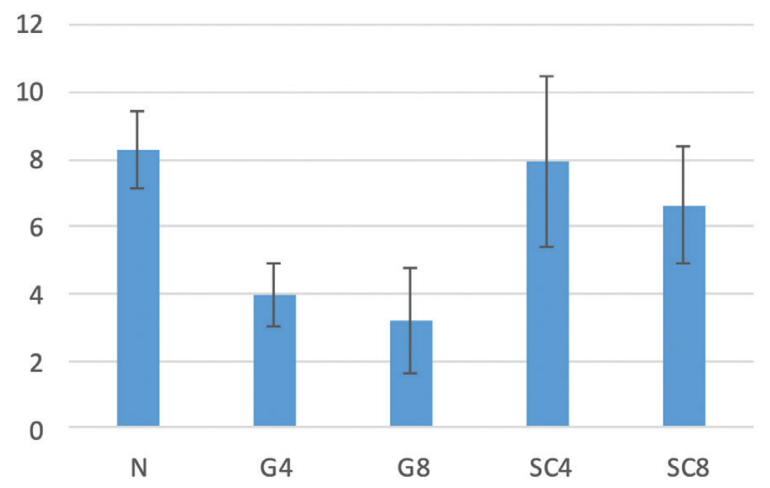

Fig. 4 Graph of mean value and standard deviation (SD) in the Osterix (Osx) expression of each group.

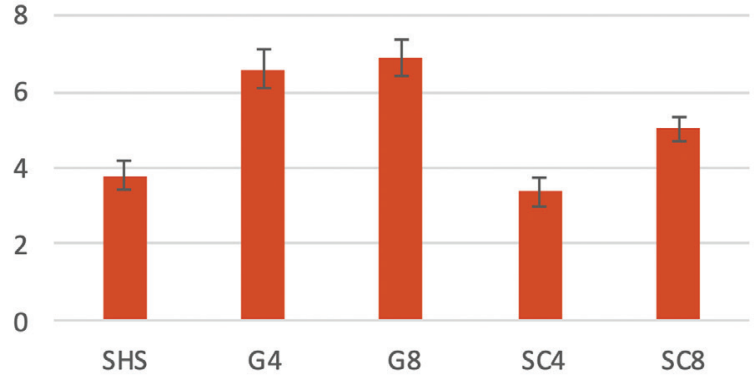

Fig. 5 Graph of mean value and standard deviation (SD) in the tartrate-resistant acid phosphatase (TRAP) expression of each group.

downstream of RUNX-2. The increase in BMP-2 and Osx expression in osteoporotic subjects, induced by hUCMSCs, shows an increase in osteoblastogenesis activity.

The increase expression in BMP-2 and Osx expression induced by hUCMSCs is in line with other studies, indicating that BMP-2 expression in the osteoporosis group is significantly lower than the normal control group. Another study showed that in the osteoporotic group, a decrease in the osteoblastogenetic process occurred, which was characterized by a lower Osx expression value than the normal control group. ${ }^{17}$ This finding is in line with those of previous studies, showing that Osx expression in the osteoporosis group was significantly lower than in the normal control groups. ${ }^{18}$

BMP-2 works in a matrix and is inactive during the homeostatic phase. The occurrence of defects in the bone triggers remodeling commencing with resorption by osteoclasts, 
accompanied by a quiescent period (silence) during which the osteoblastogenetic process begins. The increase of BMP-2 expression is caused by BMP-2 which initiates the differentiating of mesenchymal cells into preosteoblasts and toward mature osteoblasts. During this phase, the concentration, location, and expression time of the BMP-2 gene must be regulated appropriately. ${ }^{19}$

Osx will be produced by stem cells and osteoprogenitor to ensure that stem cells differentiate specifically into osteoblasts. Stem cells sourced from hUCMSCs can replace bone defects that occur with the result that it becomes a source of osteoprogenitor and osteoblasts. The increase in the number of stem cells will promote increased bone regeneration. ${ }^{20}$ Previous studies have mentioned that the high-expression of Osx after bone marrow mesenchymal stem cell (induction) will intensify osteoblastogenesis during in vivo bone regeneration. ${ }^{21}$

The decreased of TRAP expression is caused by the stem cells inhibiting osteoclast differentiation, as indicated by TRAP expression in chondroclasts, which is capable of degenerating skeletal phosphoproteins including OPN. ${ }^{12}$ TRAP is strongly expressed in osteoclasts. TRAP staining has been generally employed to identify osteoclasts and undifferentiated monocytes. TRAP is considered an important biochemical marker for osteoclasts, and its serum concentration serves as a biochemical marker of osteoclast function and the degree of bone resorption. ${ }^{22}$

A previous study demonstrated that decellularized extracellular matrix (ECM) stem cells strongly inhibit the osteoclastogenesis of primary BMMs. This inhibitory effect of ECM-derived cells on osteoclast differentiation has already been indicated by fewer TRAP-positive multinucleated cells and lower levels of osteoclast-specific gene expression. ${ }^{22}$

Labeling of PKH 26 serves to prove the existence of hUCMSCs that are injected into the mandibular bone and enable observation of its internal distribution. The presence of hUCMSCs produce a powerful florescent green color. Strong luminescence indicates the greater presence of such cells. To date, no studies have been conducted on such a method which involves direct, local injection into the osteoporotic mandibular bone. While several similar studies have involved the local administration of MSCs, these relate to different organs.

Previous studies into rat muscles involved analysis of a crush injury, administering a local injection of MSC, confirming the presence of stem cells in the MSC injection, and their migration from the local injection to all of the subject's muscles. ${ }^{23}$ The research undertaken by Wang involves attempts to administer MSC to the distal femur, in order to promote an overall increase in the thickness of the trabecular femur compared with those in the controls. ${ }^{24}$ Several studies have shown that localized therapy will produce a strong localized response. If closely associated with osteoporosis, stem cells can be systemically administered in order that they identify and move to sites requiring improvement in a process often referred to as homing.

Certain disadvantages of this systemic use include differences in the effects of MSC in specific sites, with the result that further research is required to enable regulation of the ability of cells in vivo within the body. ${ }^{25}$ This labeling of cells indicated two important facts. The first was that exogenous hUCMSCs were obtained externally to the mandibular bone in addition to the host MSC that was indeed in the body. The second was the ability to locally migrate to the bone tissue around the injection site, with the resulting possibility of this MSC being obtained for therapy at the injection site and its surroundings.

\section{Conclusions}

Augmented BMP-2 and Osx expression in osteoporotic mice induced by hUCMSCs produced an increase in osteoblastogenetic activity. The lowest TRAP expression in osteoporotic mice induced by hUCMSCs indicated a decrease in osteoclastogenesis activity. The result was that bone regeneration therapy could be managed by the induction of hUCMSCs.

\section{Suggestion}

Researchers suggest doing research with other types of markers that are not yet studied. To obtain better results and improve the use of hUCMSCs as a degenerative disease therapy, for example, diabetes mellitus.

\section{Conflict of Interest}

None declared.

\section{Acknowledgment}

This research was supported by the Stem Cell Research and Development Center, Universitas Airlangga.

\section{References}

1 Bandela V, Munagapati B, Karnati RK, Venkata GRS, Nidudhur SR. Osteoporosis: its prosthodontic considerations a review. J Clin Diagn Res 2015;9(12):ZE01-ZE04

2 Alkhader M, Aldawoodyeh A, Abdo N. Usefulness of measuring bone density of mandibular condyle in patients at risk of osteoporosis: a cone beam computed tomography study. Eur J Dent 2018;12(3):363-368

3 Abraham A, Pullishery F. The effect of menopause on the periodontium - a review. IOSR J Dent Med Sci Ver I 2015;14(4): $79-82$

4 Elani HW, Starr JR, Da Silva JD, Gallucci GO. Trends in dental implant use in the U.S., 1999 - 2016, and Projections to 2026. J Dent Res 2018;97(13):1424-1430

5 Parithimarkalaignan S, Padmanabhan TV. Osseointegration: an update. J Indian Prosthodont Soc 2013;13(1):2-6

6 Durão SF, Gomes PS, Silva-Marques JM, et al. Bone regeneration in osteoporotic conditions: healing of subcritical-size calvarial defects in the ovariectomized rat. Int J Oral Maxillofac Implants 2012;27(6):1400-1408

7 Han YF, Tao R, Sun TJ, Chai JK, Xu G, Liu J. Optimization of human umbilical cord mesenchymal stem cell isolation and culture methods. Cytotechnology 2013;65(5):819-827

8 Hendrijantini N, Kusumaningsih T, Rostiny R, Mulawardhana P, Danudiningrat CP, Rantam FA. A potential therapy of human umbilical cord mesenchymal stem cells for bone regeneration on osteoporotic mandibular bone. Eur J Dent 2018;12(3):358-362

9 Hendrijantini N, Hartono P, Ari MDA, Rantan FA. Human umbilical cord mesenchymal stem-cell therapy to increase the density of osteoporotic mandibular bone. Eur J Dent 2019;13(1):58-63 
10 Chen PM, Lin CH, Li NT, et al. c-Maf regulates pluripotency genes, proliferation/self-renewal, and lineage commitment in ROS-mediated senescence of human mesenchymal stem cells. Oncotarget 2015;6(34):35404-35418

11 Zhang C, Yuchi H, Sun L, Zhou X, Lin J. Human amnion-derived mesenchymal stem cells protect against UVA irradiation-induced human dermal fibroblast senescence, in vitro. Mol Med Rep 2017;16(2):2016-2022

12 Hayman AR. Tartrate-resistant acid phosphatase (TRAP) and the osteoclast/immune cell dichotomy. Autoimmunity 2008;41(3):218-223

13 Khajuria DK, Razdan R, Mahapatra DR. Description of a new method of ovariectomy in female rats. Rev Bras Reumatol 2012;52(3):462-470

14 Kelp A, Abruzzese T, Wohrle S, Frajs V, Aicher WK. Labeling mesenchymal stromal cells with pkh26 or vibrant dii significantly diminishes their migration, but does not affect their viability, attachment, proliferation and differentiation capacities. J Tissue Sci Eng 2017;8(2):199 doi: 10.4172/ 2157-7552.1000199

15 Kitajima Y, Doi H, Ono Y, et al. Estrogen deficiency heterogeneously affects tissue specific stem cells in mice. Sci Rep 2015;5(12861) :12861

16 Sözen T, Özışık L, Başaran NÇ. An overview and management of osteoporosis. Eur J Rheumatol 2017;4(1):46-56

17 Liu DB, Sui C, Wu TT, Wu LZ, Zhu YY, Ren ZH. Association of bone morphogenetic protein (BMP)/Smad signaling pathway with fracture healing and osteogenic ability in senile osteoporotic fracture in humans and rats. Med Sci Monit 2018;24:4363-4371

18 Mohamad N, Nabih ES, Zakaria ZM, Nagaty MM, Metwaly RG. Insight into the possible role of miR-214 in primary osteoporosis via osterix. J Cell Biochem 2019;120(9):15518-15526

19 Rogers MB, Shah TA, Shaikh NN. Turning bone morphogenetic protein 2 (BMP 2) on and off in mesenchymal cells. J Cell Biochem 2015;116(10):2127-2138

20 Sinha KM, Zhou X. Genetic and molecular control of osterix in skeletal formation. J Cell Biochem 2013;114(5):975-984

21 Tu Q, Valverde P, Li S, Zhang J, Yang P, Chen J. Osterix overexpression in mesenchymal stem cells stimulates healing of critical-sized defects in murine calvarial bone. Tissue Eng 2007;13(10):2431-2440

22 Li M, Chen X, Yan J, et al. Inhibition of osteoclastogenesis by stem cell-derived extracellular matrix through modulation of intracellular reactive oxygen species. Acta Biomater 2018;71:118-131

23 Winkler T, von Roth P, Matziolis G, Mehta M, Perka C, Duda GN. Dose-response relationship of mesenchymal stem cell transplantation and functional regeneration after severe skeletal muscle injury in rats. Tissue Eng Part A 2009;15(3):487-492

24 Wang J, Rao S, Chu J, et al. A protein interaction network for pluripotency of embryonic stem cells. Nature 2006;444(7117): 364-368

25 Antebi B, Pelled G, Gazit D. Stem cell therapy for osteoporosis. Curr Osteoporos Rep 2014;12(1):41-47 